To appear in: Erkenntnis, 2012

\title{
On Witness-discernibility of Elementary Particles
}

\author{
Øystein Linnebo ${ }^{1} \&$ F.A. Muller ${ }^{2}$
}

\begin{abstract}
In the context of discussions about the nature of 'identical particles' and the status of Leibniz's Principle of the Identity of Indiscernibles in Quantum Mechanics, a novel kind of physical discernibility has recently been proposed, which we call witnessdiscernibility. We inquire into how witness-discernibility relates to known kinds of discernibility. Our conclusion will be that for a wide variety of cases, including the intended quantum-mechanical ones, witness-discernibility collapses extensionally to absolute discernibility, that is, to discernibility by properties.
\end{abstract}

\section{Introduction}

There is a well-entrenched judgement in physics and in philosophy of physics that according to Quantum Mechanics (QM), physical systems composed of 'identical particles' consist of indiscernible objects, and therefore violate Leibniz's Principle of the Identity of Indiscernibles (PII); see French \& Krause (2008) and references therein. This judgement has recently been scrutinised, criticised and discussed in a sequence of papers, e.g. Saunders (2003), (2006), Muller \& Saunders (2008), Muller \& Seevinck (2009), Jantzen (2011), Arenhart (2012), Caulton \& Butterfield (2012). The critical argument essentially is that not just properties but also relations can discern objects, and the accompanying constructive argument is that in QM physically significant relations can be found that demonstrably discern particles. Recently Ladyman \& Bigaj (2010) have criticised the constructive argument by challenging the philosophical relevance of the employed notions of relational discernibility in the demonstrations; they propose an alternative notion of relational discernibility which does not always discern the particles, and which, they argue, is better motivated philosophically. The purpose of this brief paper is to establish that in

${ }^{1}$ Birkbeck College, London, United Kingdom; o.linnebo@bbk.ac.uk. ${ }^{2}$ Faculty of Philosophy, Erasmus University Rotterdam, and Dept. of Physics, Utrecht University, The Netherlands; f.a.muller@uu.nl . 
a wide variety of cases, the intended ones concerning QM included, Ladyman \& Bigaj's proposed new kind of relational discernibility collapses to the old notion of discernibility by properties.

In order to explain Ladyman \& Bigaj's proposal and to facilitate the demonstrations of our results, we quickly rehearse some salient terminology. We consider a physical system composed of elementary particles that share all their physical properties, such as mass, electric charge, spin, etc. — the so-called super-selected physical magnitudes. Two particles of a composite system that differ with regard to at least one of their physical properties are called absolutely discernible. We express QM in some 1st-order language $(\mathcal{L})$, that has particle-variables: $u, v, w, x, y, z$ (and labelled versions). Common practice in QM is to label particles when considering an $N$-particle system; then $\mathcal{L}$ is enriched with $N$ names $(1,2,3, \ldots, N)$ and we speak of $\mathcal{L}^{*}$. We can express absolute discernibility in $\mathcal{L}$ (and $\mathcal{L}^{*}$ ) by the following schema: particles $a$ and $b$ are absolutely discernible in QM iff (if and only if) there is a monadic open sentence $\phi(\cdot)$ in $\mathcal{L}$ (i.e. a formula in the logician's sense, with a single free variable) that holds in QM for $a$ and not for $b$ :

$$
\phi(a) \wedge \neg \phi(b) .
$$

(The asymmetry of our definition is apparent only, because the roles of $a$ and $b$ can be reversed by replacing $\phi(\cdot)$ with $\neg \phi(\cdot)$.) Particle $b$ is an individual iff it is absolutely discernible from all other particles; its 'individuality' then is expressed by the sentence in $\mathcal{L}$ that holds only for it. Particles $a$ and $b$ are relationally discernible in QM iff there is a polyadic open sentence $\rho(\cdot, \ldots, \cdot)$ in $\mathcal{L}$ such that:

$$
\exists x_{1}, \ldots, \exists x_{n}: \neg\left(\rho\left(a, x_{1}, \ldots, x_{n}\right) \longleftrightarrow \rho\left(b, x_{1}, \ldots, x_{n}\right)\right) .
$$

Particle $a$ is a relational iff it is relationally but not absolutely discernible from all other particles; and $a$ is indiscernible iff there are other particles from which $a$ is neither absolutely nor relationally discernible. In the current context, PII says that, if particles $a$ and $b$ are indiscernible, then $a=b$. If PII holds in QM, then it is a QM-impossibility for there to be indiscernible particles; so if there are indiscernibles in some model of QM, then PII is in conflict with QM.

Quine (1976) demonstrated, against a background of 1st-order classical logic, that there are two main kinds of relational discernibility. Two objects $a$ and $b$ are said to be relatively discernible iff there is a formula $\sigma(\cdot, \cdot)$ such that $\sigma(a, b)$ but not $\sigma(b, a)$. Next, $a$ and $b$ are weakly discernible iff there is a formula $\sigma(\cdot, \cdot)$ such that $\sigma(a, b)$ but not $\sigma(a, a)$. Both absolute and relative discernibiliy imply weak discernibility - for the afore-mentioned, let $\sigma(a, b)$ be the formula displayed in (1) - , but the converse implications fail.

We now say that $u$ and $v$ are physically discernible (in either of the above senses) iff the discerning sentence is physically significant. What makes a sentence in $\mathcal{L}$ physically significant? Muller \& Saunders (2008) answer generally by imposing two requirements: (Req1) it is defined in terms of physical magnitudes (spin, energy, position, momentum, \&c.); and (Req2) it respects the symmetries of the theory, which is in the case of QM 
permutation and (Galilean) symmetry. The Symmetrisation Postulate of QM applies to physical systems consisting of particles that are not absolutely discernible. Only relational discernibility then is permitted by QM. Req2 imposes a further restriction: only weak relational discernibility is permitted by QM, because the only permutation-invariant binary relations are symmetric ones. Muller \& Saunders (2008) demonstrate that in QM, fermions are weakly discernible by means of relations, some of which can be interpreted in terms of spin (thereby meeting the two Requirements); and Muller \& Seevinck (2008) demonstrate that in QM, all elementary particles, fermions and bosons included, are weakly discernible by means of the canonical commutator relation, which is a symmetric relation defined in terms of linear momentum and position (thereby meeting the two Requirements).

Ladyman \& Bigaj (2010) adhere to the above terminology. They judge physical weak discernibility however too weak to qualify as an acceptable concept of physical discernibility, because it fails to say which particle is which. Ladyman \& Bigaj then write (2010: 128-9; our emphasis):

This is not to say that the entire category of relational discernibility has to be rejected as nonphysical and that the only admissible discernibility is the absolute one. Let us make this clear by considering a perfectly acceptable case of relational discernibility not reducible to the absolute one. [...] [W] should make sure that there is a proper physical procedure that could in principle identify those objects as distinct from the remainder of the universe that does not discern them. Hence, we suggest that a corrected version of the condition of relational discernibility should look something like that:

$a$ and $b$ are relationally physically discernible in $\mathcal{L}$ iff there is some physically defined relation $R$ such that $\exists x[(\operatorname{Rax} \wedge \neg R b x) \wedge \forall y(y$ Ind $x \rightarrow($ Ray $\wedge \neg R b y))]$, where ' $y$ Ind $x$ ' means that $y$ is absolutely physically indiscernible from $x$.

This passage makes it clear that their goal is to articulate a new kind of relational discernibility which is better motivated philosophically than the standard notion of weak discernibility, yet is not extensionally equivalent to (or 'reducible to', as Ladyman \& Bigaj put it) absolute discernibility, or to any of the other mentioned kinds of discernibility mentioned above. A somewhat similar idea was advanced by Dieks \& Versteegh (2008: 933), who call witness $x$ a 'gauge system'; they argue against the appeal to such a 'gauge system' in order to discern particles that are not absolutely discernible, in terms of expectationvalues, but they do not propose a definition of a new kind of discernibility to make this idea explicit.

We claim that witness-discernibility turns out not to be a novel discernibility category that differs extensionally from existing kinds. In Section 2, we show that, for a wide variety of cases, witness-discernibility coincides extensionally with absolute discernibility. In the final Section 3, we draw conclusions and take a walk on an avenue of escape for proponents of witness-discernibility. The only option left that might be viable, we argue, is to locate the value of the notion of witness-discernibility wholly at the intensional level, as a new and interesting route to a well known relation-in-extension. 


\section{Equivalence Theorems}

We now wish to establish some general logico-mathematical results whose validity is not limited to QM. So we work in the general setting provided by 1st-order logic and its model theory (for more examples of this approach, see Ladyman, Linnebo, and Pettigrew (2012)).

Consider a 1st-order language $\mathcal{L}$ with a model $\mathcal{M}$. Let $a$ and $b$ be objects in the domain of $\mathcal{M}: a, b \in \operatorname{dom}(\mathcal{M})$.

Definition 1 (a) Objects $a$ and $b$ are absolutely discernible in $\mathcal{M}$ iff there is a monadic open sentence $\phi(\cdot)$ in $\mathcal{L}$ that is satisfied by $a$ but not by $b: \mathcal{M} \models \phi(a) \wedge \neg \phi(b)$.

(b) Objects $a$ and $b$ are witness-discernible in $\mathcal{M}$ iff there is a dyadic open sentence $\psi(\cdot, \cdot)$ in $\mathcal{L}$ and an object $c \in \operatorname{dom}(\mathcal{M})$, which we call a witness, such that:

(i) $\mathcal{M} \models \psi(a, c) \wedge \neg \psi(b, c)$, and

(ii) For any $d \in \operatorname{dom}(\mathcal{M})$ not absolutely discernible in $\mathcal{M}$ from $c$ : $\mathcal{M} \models \psi(a, d) \wedge \neg \psi(b, d)$.

We believe our talk about there being a dyadic open sentence $\psi(x, y)$ in $\mathcal{L}$ to be a reasonable explication of Ladyman \& Bigaj's talk about a "physically defined relation $R$ " (129). Moreover, we observe that clause (i) is in fact redundant, as it follows from clause (ii) and the observation that $c$ is not absolutely discernible from itself: choose $c$ for $d$ in (ii) and (i) obtains.

Since we already defined what absolute discernibility is in Section 1, have we not defined it twice over? In fact, two subtly different notions have been defined. In Section 1, we have defined absolute discernibility simpliciter, whereas we have now defined absolute discernibility in a model. The afore-mentioned notion corresponds to a special case of the last-mentioned where the model is chosen to represent the actual world. (Similarly, truth simpliciter corresponds to truth in a model representing the actual world.)

We now proceed to some theorems that relate the notions of witness-discernibility and absolute discernibility under various conditions. No conditions are present in the first theorem.

Theorem 1 In every model $\mathcal{M}$ of $\mathcal{L}$, any two elements in $\operatorname{dom}(\mathcal{M})$ that are absolutely discernible are also witness-discernible.

Proof. Suppose $a$ and $b$ are absolutely discernible by monadic open sentence $\rho$ of $\mathcal{L}$ :

$$
\mathcal{M} \models \rho(a) \wedge \neg \rho(b) .
$$

To show that $a$ and $b$ are also witness-discernible, we must find a dyadic open sentence of $\mathcal{L}$, say $\psi(\cdot, \cdot)$, such that there is some $c \in \operatorname{dom}(\mathcal{M})$ such that for any $d \in \operatorname{dom}(\mathcal{M})$ that is not absolutely discernible from $c$ in $\mathcal{M}$ :

$$
\mathcal{M} \models \psi(a, d) \wedge \neg \psi(b, d) .
$$


One easily verifies that, due to (3), the following sentence qualifies:

$$
\rho(x) \vee(\varphi(y) \vee \neg \varphi(y))
$$

where $\varphi(\cdot)$ is any monadic open sentence. Q.e.d.

Theorem 2 Whenever $\mathcal{L}$ allows infinite conjunctions, any two objects are absolutely discernible in model $\mathcal{M}$ of $\mathcal{L}$ iff they are witness-discernible in $\mathcal{M}$.

Proof. The sufficiency of absolute discerniblity for witness discernibility follows from Theorem 1, which holds for any language $\mathcal{L}$, including infinitary ones. Next the necessity. Premise: $a$ and $b$ are witness-discernible in $\mathcal{M}$ with witness $c$ and $\psi(\cdot, \cdot)$ as in Definition 1. Let $\Phi(x)$ be the conjunction of open sentences $\phi(x)$ such that $\mathcal{M} \models \phi(c)$ :

$$
\bigwedge_{\mathcal{M} \models \phi(c)} \phi(x) .
$$

Note that $\mathcal{M} \models \Phi(d)$ iff $c$ and $d$ are not absolute discernible. Let $\chi(x)$ be the following sentence:

$$
\exists y(\Phi(y) \wedge \psi(x, y))
$$

Then we have $\mathcal{M} \models \chi(a)$, because $c$ serves as a witness to the existence claim. But we cannot have $\mathcal{M} \models \chi(b)$; for if we did, then $\mathcal{M}$ would contain an object $d$, not absolutely discernible from $c$, such that $\mathcal{M} \models \psi(b, d)$, in violation of clause (ii) of Definition 1 (b) of witness-discernibility. Thus $\mathcal{M} \forall \chi(b)$ and $\mathcal{M} \models \chi(a)$, which is to say that $\chi$ absolutely discerns $a$ and $b$ in $\mathcal{M}$. Q.e.d.

Theorem 3 If the domain of $\mathcal{M}$ is finite, then any two elements are absolutely discernible in $\mathcal{M}$ iff they are witness-discernible.

Proof. By Theorem 1, absolute discernibility is sufficient for witness-discernibility in general and thus a fortiori in models with finite domains.

Now for the necessity. Since the domain is finite, we may assume that $\mathcal{L}$ is finitary; no new expressive power would be gained by allowing infinite conjunctions. Choose some enumeration $\left\{\phi_{j}(x) \mid j \in \omega\right\}$ of the sentences of $\mathcal{L}$ with $x$ as the only free variable. We want a sentence $\psi(x)$ such that $\mathcal{M} \models \psi(d)$ iff $c$ and $d$ are not absolute discernible. Consider finitary approximations $\Phi_{n}(x)$ of the infinite conjunction $\Phi(x)$ used in the proof of Theorem 2, defined as:

$$
\bigwedge_{\mathcal{M}=\phi_{j}(c), 0 \leqslant j \leqslant n} \phi_{j}(x) .
$$

We claim that there is an $n$ such that $\Phi_{n}(x)$ and $\Phi(x)$ have the same extension (•). If so, then $\Phi_{n}(x)$ can be used instead of $\Phi(x)$ in the proof of Theorem 2, and we are done. Assume, for reductio, that claim $(\bullet)$ fails. Then the $\Phi_{n}(x)$ would form an infinite sequence 
of sentences whose extensions get strictly less inclusive for arbitrarily large $n$. But this cannot happen in a finite domain. So claim $(\bullet)$ stands. Q.e.d.

Theorem 3 raises the question whether witness-discernibility and absolute discernibility come apart in infinite models. By Theorem 1, in any model, two absolute discernible objects are witness-discernible. The next Theorem refutes the converse in denumerably infinite domains.

Theorem 4 When $\mathcal{L}$ is finitary, and specifically does not permit infinite conjunctions, then there are models with a denumerably infinite domain that contains objects that are witness-discernible but not absolute discernible.

Proof. Let $\mathcal{L}$ be a language with just two primitive predicates: $S(x, y)$ (for ' $x$ sees $y$ ') and $T(x, y)$ (for ' $x$ touches $y^{\prime}$ ). Let $C^{n}(x)$, for $n \geqslant 1$, abbreviate:

$$
\exists x_{1}, \exists x_{2}, \ldots, \exists x_{n}: T\left(x, x_{1}\right) \wedge T\left(x_{1}, x_{2}\right) \wedge \ldots \wedge T\left(x_{n-1}, x_{n}\right) ;
$$

that is, that $x$ participates in a chain of at least $n$ objects (possibly with repetitions) each of which touches the next. We now describe a denumerably infinite model $\mathcal{M}^{\omega}$ of $\mathcal{L}$. $\operatorname{Dom}\left(\mathcal{M}^{\omega}\right)$ contains two objects, which we call Castor and Pollux, that are not absolutely discernible. For every $n$, both can see an object, call it $c_{n} \in \operatorname{dom}\left(\mathcal{M}^{\omega}\right)$, that is the initial member of a finite chain, of exactly $n$ objects, each of which touches the next:

$$
\mathcal{M}^{\omega} \models C^{n}\left(c_{n}\right) \wedge S\left(\text { Castor }, c_{n}\right) \wedge S\left(\text { Pollux }, c_{n}\right) .
$$

But only Castor can see an object, $c \in \operatorname{dom}\left(\mathcal{M}^{\omega}\right)$, that is the initial member of a chain of a denumerable infinity of objects, each of which touches the next:

$$
\mathcal{M}^{\omega} \models S(\text { Castor }, c) \wedge \neg S(\text { Pollux }, c) .
$$

There are no other objects in the domain, and no other relations obtain.

We claim that in $\mathcal{M}^{\omega}$, Castor and Pollux are witness-discernible. Clearly (9) is clause (i) of witness-discernibility. In order to verify clause (ii), it suffices to verify that object $c$ is absolutely discernible from every other object of $\operatorname{dom}\left(\mathcal{M}^{\omega}\right)$. Let $d \in \operatorname{dom}\left(\mathcal{M}^{\omega}\right)$ be distinct from $c$. Then there is some $m$ such that $d$ is not an initial member of a chain of at least $m$ objects each of which touches the next. This ensures that there is a monadic sentence that distinguishes $c$ from $d$, which makes $c$ and $d$ absolutely discernible.

It remains to show that Castor and Pollux are not absolutely discernible in $\mathcal{M}^{\omega}$. This is easily proved by induction on the complexity of sentences of $\mathcal{L}$, bearing in mind that $\mathcal{L}$ is finitary and so does not permit infinite conjunctions. Q.e.d.

In (textbooks and papers on) QM, particles are labelled as a matter of course when considering physical systems composed of particles that are not absolutely discernible. Hence let us extend $\mathcal{L}$ with names, resulting in $\mathcal{L}^{*}$, which has a name for each element of 
$\operatorname{dom}(\mathcal{M})-1,2,3, \ldots(N)$. In models of $\mathrm{QM}$, the number of particles is finite. Logically we can consider a denumerably infinite number of particles, in which case $\mathcal{L}^{*}$ has a denumerably infinity of names.

Theorem 5 In every model $\mathcal{M}^{*}$ of $\mathcal{L}^{*}$, any two elements in $\operatorname{dom}\left(\mathcal{M}^{*}\right)$ are witness-discernible iff they are absolutely discernible.

Proof. The sufficiency of absolute discernibility for witness-discernibility follows from Theorem 1, which holds for every language, including ones with names. We turn to the necessity.

Premise: 1 and 2 are witness-discernible in model $\mathcal{M}^{*}$. Then we have some dyadic open sentence, say $\psi(\cdot, \cdot)$, such that there is some $c \in \operatorname{dom}\left(\mathcal{M}^{*}\right)$, which has a name in $\mathcal{L}^{*}$ : call it $\bar{c}$, such that for any $d \in \operatorname{dom}\left(\mathcal{M}^{*}\right)$, which also has a name $(\bar{d})$, if $d$ is not absolutely discernible from $c$ in $\mathcal{M}^{*}$, then:

$$
\mathcal{M}^{*} \models \psi(1, \bar{d}) \wedge \neg \psi(2, \bar{d}) .
$$

To show that 1 and 2 are also absolutely discernible in $\mathcal{M}^{*}$, we must find a monadic open sentence, say $\rho(\cdot)$, such that $\mathcal{M} \models \rho(1) \wedge \neg \rho(2)$. One easily verifies that this is such a sentence (having free variable $x$ ):

$$
\psi(x, \bar{c}) \wedge \neg \psi(2, \bar{c}) .
$$

Q.e.d.

Notice that without names, this proof is no good: sentence (11) would be an open sentence having three variables rather than one.

Theorem 5 is in harmony with a result of Ladyman, Linnebo, \& Pettigrew (2012) that, in $\mathcal{L}^{*}$, weak discernibility entails absolute discernibility. For we also know that in any language, absolute discernibility implies witness-discernibility, which in turn implies weak discernibility (Section 1). By closing the loop, it follows that the four forms of discernibility in $\mathcal{L}^{*}$ (weak, relative, witness-discernibility and absolute) are extensionally equivalent.

\section{Discussion and Conclusions}

Our theorems show that witness-discernibility collapses to absolute discernibility in most cases of interest.

By Theorem 3, one assumption sufficient for this collapse is that the domain is finite. This is physically very encompassing, because physical theories describing composite physical systems consisting of particles that are not absolutely discernible always consist of a finite number of particles. Ladyman \& Bigaj (2010: 124) also call a finite domain "a plausible assumption for physical objects". Systems of a denumerable infinitude of particles have been considered, but rarely, and they arguably are not instantiated in our 
physical universe. (Fields instantiate an infinitude of degrees of freedom; fields, classical and quantum, are absolutely discernible and therefore discernible in all other ways.) By Theorem 4, the only case where absolute discernibility and witness-discernibility may come apart, in that we have witness-discernible objects that are not absolutely discernible, is where we have infinitely many objects. Another case of physical interest is space-time, where we have, on a substantivalist reading, a non-denumerable infinitude of objects (i.e. space-time points). Perhaps in such cases the mentioned notions also come apart. We have no theorems to report on this issue, but we do want to point out the following.

In symmetric space-times of the General Theory of Relativity, all points in a 3-dimensional hypersurface (a leaf of the foliation of space-time) are weakly but not absolutely discernible, which implies that PII holds (see Muller (2011)). But these points are not witnessdiscernible (as a moment's reflection will reveal), so that if weakly discerning relations do not really discern, as Ladyman \& Bigaj maintain, and have to be replaced with witness discerning relations in the sufficient condition for identity (PII), then PII will entail the absurd conclusion that there is only a single space-time point in a 3-dimensional hypersurface. Thus, if PII is formulated in terms of witness-discernibility, its validity will be more restricted than one would have thought.

By Theorem 2, another sufficient condition for the collapse of witness-discernibility to absolute discernibility is that the language contains infinite conjunctions. In the event that we want to consider infinitely many objects, it seems plausible to allow for infinite conjunctions in one's definitions of physical properties and relations.

By Theorem 1, without any restrictions on the size of the domain or on whether $\mathcal{L}$ is finitary or infinitary, absolutely discernible objects always are also witness-discernible. By Theorem 5, the converse also holds provided the objects in the model bear names, such as when particles are labelled - which thus is another sufficient condition for the mentioned collapse.

Let us finally consider whether the case for witness-discernibility can be improved by modifying the definition of the new form of relational discernibility by requiring that the witness be distinct from each of the two objects to be discerned. Ladyman \& Bigaj write in the displayed quotation in Section 1 that "there is a proper physical procedure that could in principle identify those objects as distinct from the remainder of the universe that does not discern them." Clearly the remainder of the universe is what remains when we have set the candidate objects for discerning apart. The witnessing object $c$ belongs to that remainder, so that one must have that $a \neq c \neq b$. To express this, we need to work in a language with an identity predicate. Let $\mathcal{L}=$ be such a language. Then we define that $a$ and $b$ are witness ${ }^{+}$-discernible in model $\mathcal{M}$ of $\mathcal{L}=$ iff there is an open sentence $\psi(x, y)$ in $\mathcal{L}$ and an object $c \in \operatorname{dom}(\mathcal{M})$ such that:

(i) $\mathcal{M} \models \psi(a, c) \wedge \neg \psi(b, c) \wedge a \neq c \neq b$, and

(ii) for any $d \in \operatorname{dom}(M)$ not absolutely discernible in $\mathcal{M}$ from $c$ and distinct from $a$ and $b$, 
we have: $\mathcal{M} \models \psi(a, d) \wedge \neg \psi(b, d)$.

However, this modification provides no solace for defenders of witness-discernibility. For we easily see that, for any model $\mathcal{M}$ and language with identity $\mathcal{L}^{=}$, absolute discernibility entails witness ${ }^{+}$-discernibility, which in turn entails witness-discernibility. But we have identified physically plausible conditions under which witness-discernibility entails absolute discernibility. By completing the circle of entailments, it follows that, under these conditions, witness ${ }^{+}$-discernibility too collapses to absolute discernibility.

Theorem 5 employs names, in opposition to the prohibition of names by Ladyman \& Bigaj (2010: 124). The motivation to forbid names is that they permit us to discern the particles by using no more than the fact that they bear names: that is too cheap. We agree. What we like to point out however is that forbidding names is a kill or cure remedy. How to state the Symmetrisation Postulate of QM without labelling the particles? Here names are employed to express a crucial assumption of QM about physical systems consisting of particles that are not absolutely discernible. There is nothing against labelling (naming) the particles; specifically, there is nothing against expressing physically significant propositions by using names, e.g. the Symmetrisation Postulate. What happens in the proof of Theorem 5 is that we begin by assuming that particles 1 and 2 are witness-discernible, and then show they are also absolutely discernible by defining a monadic predicate that is defined in terms of the witness discerning dyadic predicate: if the last-mentioned is physically significant, then so is the afore-mentioned. The use of names does not obliterate the physical significance of a proposition. Their presence in the proof of Theorem 5 is unobjectionable.

We conclude that witness-discernibility is extensionally equivalent to absolute discernibility on each of a number of plausible assumptions; notably, in the case of $N$-particle systems in QM, the case of interest, the collapse is guaranteed. Any philosophical interest of the notion of witness-discernibility would thus have to lie at the intensional level. (This is how Ladyman \& Bigaj (2011) wish to respond.) For even in cases of extensional equivalence with absolute discernibility, it cannot be denied that witness-discernibility provides a new route to what turns out to be a well known relation-in-extension; an unexpected meaning can indeed be ascribed to 'absolute discernibility'. We leave for others to assess the philosophical value of this new intensional route to a familiar extension.

\section{Acknowledgments.}

We want to thank two anonymous referees for some helpful remarks, and J. Ladyman, T. Bigaj and the members of the Bristol Structuralism Seminar for conversations on the subject-matter. Øystein Linnebo was supported by an ERC Starting Grant.

\section{References}

Arenhart, J.R.B. (2012), 'Many Entities, No Identity', to appear in: Synthese.

Caulton, A., Butterfield, J. (2012), ‘On Kinds of Indiscernibility in Logic and Metaphysics', British 
Journal for the Philosophy of Science 63, 27-84.

Dieks, D., Versteegh, M.A.M. (2008), 'Identical Quantum Particles and Weak Discernibility', Foundations of Physics 38: 923-934.

French, S., Krause, D. (2006), Identity in Physics: A Historical, Philosophical and Formal Analysis, Oxford: Clarendon Press.

Jantzen, B.C. (2011), 'No Two Entities Without Identity', Synthese 181: 433-450.

Ladyman, J., Bigaj, T. (2010), 'The Principle of the Identity of Indiscernibles and Quantum Mechanics', Philosophy of Science 77: 117-136.

Ladyman, J., Bigaj, T. (2011), private communication by e-mail, 2011.

Ladyman, J., Linnebo, Ø., Pettigrew, R. (2012), 'Identity and Discernibility in Philosophy and Logic', Review of Symbolic Logic 5.1: 162-86

Muller, F.A. (2011), 'How to Defeat Wüthrich's Abysmal Embarrassment Argument against SpaceTime Structuralism', Philosophy of Science 78: 1046-1057.

Muller, F.A., Saunders, S.W. (2008), 'Discerning Fermions', British Journal for the Philosophy of Science 59: 499-548.

Muller, F.A., Seevinck, M.P. (2009), ‘Discerning Elementary Particles', Philosophy of Science 76: 179_ 200.

Quine, W.v.O. (1976), 'Grades of Discriminability', Journal of Philosophy 73: 113-116.

Saunders, S. (2003), 'Physics and Leibniz's Principles', in: K. Brading, E. Castellani (eds.), Symmetries in Physics: Philosophical Reflections, Cambridge: Cambridge University Press, 2003: 289307.

Saunders, S. (2006), 'Are quantum particles objects?', Analysis 66.1: 52-63. 